\title{
Balanced Electrolyte Solutions or Normal Saline? Resuscitative Fluid Administration Practice in Swiss Pediatric Acute Care A Cross-Sectional Study
}

\author{
Jasmin L. Huber, * Steffen Berger, MD,† and Ruth M. Löllgen, MD
}

\begin{abstract}
Introduction: The ideal asanguineous intravenous fluid for volume resuscitation in children is controversially debated and clinical practice guidelines are scarce. Administration of large amounts of normal saline has been associated with complications including hyperchloremic acidosis, dysnatremia, neurologic damage, and fatality.

Aim: We examined the current practice of intravenous fluid and blood product administration in acutely ill and injured children among pediatric acute care physicians in Switzerland.

Methods: For this descriptive, cross-sectional study, pediatric emergency departments, pediatric and neonatal intensive care units were surveyed by means of an online questionnaire.

Results: Sixty of 66 departments and 47 of 87 participants returned the survey. Normal saline (NS) was most commonly administered ( $n=42$ / $46,91.3 \%$ ) and twice as many times as balanced electrolyte solutions $(\mathrm{n}=20 / 46,43.5 \%)$. The mean fluid volumes ranged from 7.9 to $19.1 \mathrm{~mL} / \mathrm{kg}$. Hypertonic saline/NS were selected most often for shock with severe head injury. Half of participants administered colloids (48.9\%). Packed red blood cells (97.7\%) and fresh frozen plasma (88.4\%) were most frequently given blood products.

Conclusion: There is a distinct practice variation in intravenous fluid and blood product administration in children in Switzerland. Although NS is most frequently given, we observed a trend toward the use of balanced electrolyte solutions. Prospective studies are warranted to compare NS with balanced electrolyte solution (BES) in the pediatric acute care setting. We suggest that pediatric fluid administration guidelines and mass transfusion protocols are implemented to standardize this frequent intervention and minimize complications.
\end{abstract}

Key Words: balanced electrolyte solutions, intravenous fluids, normal saline, resuscitation, shock, trauma

(Pediatr Emer Care 2019;00: 00-00)

\section{WHAT IS ALREADY KNOWN ON THIS SUBJECT}

There is growing evidence that BES might be preferable to NS for fluid resuscitation in the acute setting. However, these data mainly stem from adult studies and current intravenous fluid administration practice in pediatric patients is inconsistent and not evidence based.

From the *Faculty of Medicine, University of Bern; and $\dagger$ Department of Pediatric Surgery, and $\ddagger$ Pediatric Emergency Department, Inselspital, University Hospital, University of Bern, Bern, Switzerland.

Disclosure: The authors declare no conflict of interest

Reprints: Ruth M. Löllgen, MD, Pediatric Emergency Department, Inselspital,

University Hospital, University of Bern, Freiburgstrasse 10, 3010 Bern, Bern, Switzerland (e-mail: ruth.loellgen@gmail.com).

Supplemental digital content is available for this article. Direct URL citations appear in the printed text and are provided in the HTML and PDF versions of this article on the journal's Web site (www.pec-online.com).

Copyright $₫ 2019$ Wolters Kluwer Health, Inc. All rights reserved.

ISSN: 0749-516

\section{WHAT THIS STUDY ADDS}

Our study reveals a distinct clinical practice variation in intravenous fluid resuscitation among pediatric acute care physicians in Switzerland. The observed overall trend toward the use of BES is in contrast to a rift between West Switzerland, still preferring NS, and East Switzerland, tending to use BES. In combination with dissention on this topic in the literature and lack of evidence in children, our findings emphasize the need for prospective studies determining the benefits and adverse effects of various crystalloids in pediatric patients.

\section{INTRODUCTION}

Intravenous (IV) fluid administration is a lifesaving cornerstone of therapy in critically ill children but may evoke metabolic complications including dysnatremia, hyperchloremic metabolic acidosis, hyponatremic encephalopathy, permanent neurological damage, and death. ${ }^{1}$ The ideal asanguineous IV fluid is controversially debated. Isotonic fluids protect against dysnatremia ${ }^{1}$ and therefore are the fluid of choice for initial volume resuscitation. ${ }^{1-3}$ Normal saline (NS) is most frequently used for fluid resuscitation. ${ }^{2,4}$ However, by using large volumes of NS containing supraphysiologic chloride $(\mathrm{Cl})$ contents $(154 \mathrm{mEq} / \mathrm{L})$, plasma $\mathrm{Cl}$ levels may rise excessively and induce hyperchloremic metabolic acidosis. ${ }^{2,5}$ Polyelectrolyte solutions (Lactated Ringer's [LR], Ringer acetate [RA]) contain more physiological $\mathrm{Cl}$ contents resembling human plasma $(94-111 \mathrm{mEq} / \mathrm{L}), 109 \mathrm{mEq} / \mathrm{L}$ and $98 \mathrm{mEq} / \mathrm{L}$, respectively, and are alkalinizing agents. ${ }^{5}$ Pediatric studies comparing NS to balanced electrolyte solutions (BES) are scarce and contradictory. ${ }^{6-8}$ Recently, published results suggest that fluid resuscitation with BES instead of NS in critically ill adult patients reduces adverse kidney events and fatality and increases hospital-free days. "Damage control resuscitation" in pediatric trauma advocates smaller volume resuscitation and permissive hypotension for the benefit of reducing fluid extravasation, cerebral edema, raised intracranial pressure, and damaged microvasculature. ${ }^{10}$ Only few pediatric IV fluid clinical practice guidelines $(\mathrm{CPG})^{3}$ and massive transfusion protocols (MTP) exist and plasma transfusion guidelines are not standardized. ${ }^{11,12}$ We assessed the current practice of IV fluid resuscitation and transfusion standards in critically ill children among pediatric emergency medicine physicians, pediatric intensivists, and neonatologists in Switzerland.

\section{METHODS}

\section{Study and Survey Design}

We composed a web-based questionnaire comprising 27 questions on IV fluid, blood product (BP) and tranexamic acid (TXA) administration, and use of MTPs (see Supplemental Digital Content 1, http://links.lww.com/PEC/A407). We translated the survey into standard German (63\% of Swiss residents are native speakers), 


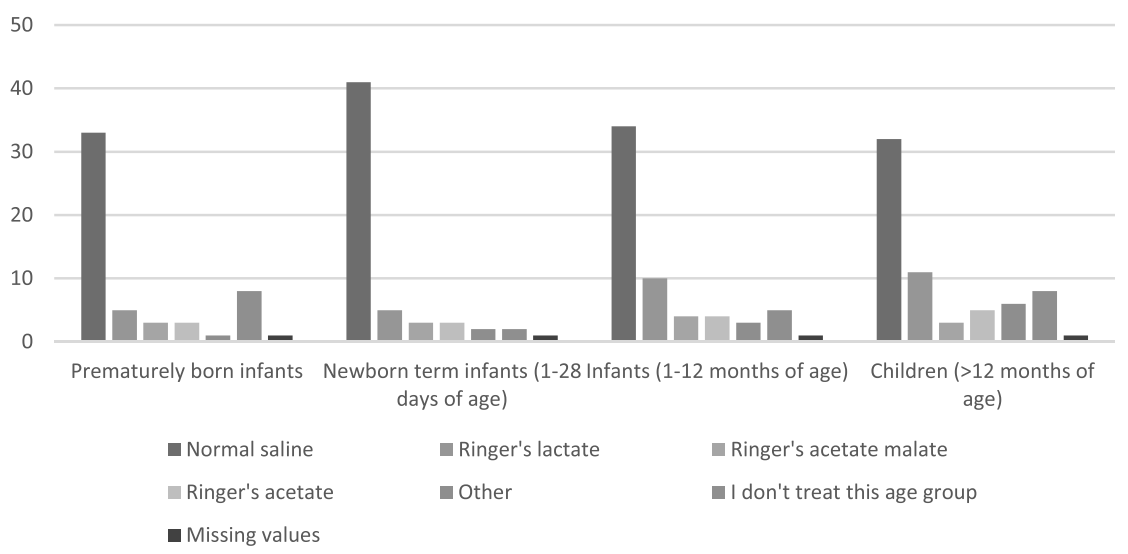

FIGURE 1. Intravenous fluid applied for all types of $\operatorname{shock}(n=47$ respondents, multiple answers were possible).

French (22.7\%), and English (for Italian [8.4\%] and Romansh $[0.6 \%]$ native speakers), validated it linguistically (see Supplemental Digital Content 2, http://links.lww.com/PEC/A408), and formatted it using Survey Monkey.

\section{Study Population}

We surveyed all Swiss pediatric intensive care units (PICUs, $n=9$ ), pediatric emergency departments (PEDs, $n=28$ ), neonatal intensive care (NICU, $\mathrm{n}=9$ ), and neonatal units (NUs, $\mathrm{n}=20$ ) on IV fluid administration and transfusion strategies, using the foederatio medicorum helveticorum register of educational hospitals for reference (http://www.siwf-register.ch). Level III, IIb, and IIa NICUs, caring for neonates aged $\geq 240 / 7$ weeks, $\geq 32$ 0/7 weeks, and $\geq 340 / 7$ weeks of gestational age, respectively, were included, and level 1 NUs were excluded (http://www.neonet.ch). We dispatched the questionnaire by e-mail to 1 senior physician of each department. Nonresponders received 2 two-weekly reminders by e-mail, and if still unanswered, questionnaires were redirected to an alternate physician of the same department.

\section{Statistical Analysis}

We examined the quantitative questions using SPSS statistics, Version 24. For yes/no questions, the proportion of physicians responding yes/no was presented as percentage. We used the Fisher exact test to evaluate the $P$ value of differences between the French- and German/Italian-speaking parts of Switzerland, differences were defined as being significant less than a $P$ value of 0.05 . For questions allowing multiple answers, we evaluated

TABLE 1. Intravenous Volume Administered According to Type of Shock

\begin{tabular}{lcc}
\hline Type of Shock & $\begin{array}{c}\text { No. } \\
\text { Observations }\end{array}$ & $\begin{array}{c}\text { IV Volume } \\
\text { Administered, mL/kg }\end{array}$ \\
\hline Anaphylactic & 40 & $16.9(5.63)$ \\
Septic & 40 & $18.4(3.5)$ \\
Hemorrhagic & 38 & $19.1(2.8)$ \\
Cardiogenic & 42 & $7.9(5.0)$ \\
Hypovolemic & 41 & $18.5(3.6)$ \\
Hypovolemic (in the & 37 & $16.5(5.8)$ \\
$\quad$ context of DKA) & 36 & $15.6(5.7)$ \\
\hline Neurogenic & 36 &
\end{tabular}

Values are mean (SD), unless otherwise indicated. the absolute number as well as percentage (therefore not summing up to 100).

\section{RESULTS}

From October to November 2016, 60 of 66 departments returned the survey, achieving a response rate of $90.1 \%$ : PED, $n=$ 12 , PICU, $\mathrm{n}=5$, NICU, $\mathrm{n}=6$, and multiple departments within 1 hospital under 1 director: NICU/PED $(n=14 \times 2)$, NICU/PICU $(\mathrm{n}=3 \times 2)$, and NICU/PICU/PED $(\mathrm{n}=1 \times 3)$. Of 87 respondents, $47(54.0 \%)$ composed $12.4 \%$ of all PICU, NICU, and PED specialists practicing in Switzerland $(\mathrm{n}=380)$ as of January 20, 2017 (see Supplemental Digital Content 3, http://links.lww.com/ PEC/A409). Because neonates may present with different pathologies than infants and children, we subdivided the respondents into 2 groups for analysis of IV fluid, BP, TXA, and MTP use: group A (PED, PICU, $n=17)$ excluded and group $B(n=30)$ included departments treating neonates. Because of our reminder style, more than 1 questionnaire was returned from the same

TABLE 2. Reasons for Choice of Colloids and Crystalloids

A. Reasons for switching to colloids after $\mathrm{n}=23$ initial use of crystalloids

Hypoalbuminemia

$16(69.6)$

Lack of clinical response

$11(47.8)$

After a fixed volume of crystalloids

$6(26.1)$

B. Reasons for using colloids rather than crystalloids

$\mathrm{n}=23$

Higher intravascular volume expansion effect

$10(43.5)$

Limitations of fluid overload

$3(13.0)$

Maintenance of colloid oncotic pressure

$12(52.2)$

Preservation of microcirculation

2 (8.7)

Other reasons

C. Reasons for using crystalloids rather than colloids

$4(17.4)$

$\mathrm{n}=47$

Costs

$20(42.6)$

Impairment of coagulation

$10(21.3)$

Risk of renal dysfunction

$13(27.7)$

No difference in efficacy $31(66.0)$

Storage/long term effects $\quad 6(12.8)$

Risk of virus/prion transmission 7 (14.9)

Other reasons

$10(21.3)$

Values are number (percentage).

Groups do not sum up to $100 \%$ because multiple answers were possible. 
TABLE 3. Type of IV Fluid Administered for Hemorrhagic Shock Before BP Application, Chronologically Classified ( $n=47$, Missing Answers: $n=4$ )

\begin{tabular}{|c|c|c|c|c|}
\hline & Quick RBCs & First choice $(n=47)$ & Second choice $(n=47)$ & Third choice $(n=47)$ \\
\hline Quick RBCs & $5(10.6)$ & & & \\
\hline NS & & $28(59.6)$ & $1(2.1)$ & 0 \\
\hline FFP & & $1(2.1)$ & $6(12.8)$ & $3(6.4)$ \\
\hline Albumin & & 0 & 0 & $4(8.5)$ \\
\hline LR & & $4(8.5)$ & $7(14.9)$ & 0 \\
\hline RA & & $2(4.3)$ & 0 & 0 \\
\hline RAM & & $1(2.1)$ & $2(4.3)$ & 0 \\
\hline Other & & $1(2.1)$ & 0 & 0 \\
\hline Missing values & $5(10.6)$ & & & \\
\hline Applied IV fluids before administration of BP & & $\geq 1$ IV fluid $(\mathrm{n}=37)$ & $\geq 2$ IV fluids $(\mathrm{n}=16)$ & $\geq 3$ IV fluids $(\mathrm{n}=7)$ \\
\hline
\end{tabular}

department in 4 cases. Because not all answers from 1 department were identical, all questionnaires were included for analysis.

\section{Intravenous Fluid and Volume}

Most respondents used NS for shock throughout all age groups $(n=42 / 46,91.3 \%)$, whereas BES were administered by fewer participants ( $n=20 / 46,43.5 \%$ ) (Fig. 1). The mean volumes for diverse shocks ranged from 15.6 to $19.1 \mathrm{~mL} / \mathrm{kg}$ per bolus; cardiogenic shock was treated by smaller volumes $($ mean $=7.9 \mathrm{~mL} / \mathrm{kg}$, range $0-20 \mathrm{~mL} / \mathrm{kg}$ ) (Table 1$)$.

\section{Colloids}

Half of participants ( $n=23 / 47,48.9 \%)$ used colloids. The most common reasons for switching to colloids after crystalloids, using colloids rather than crystalloids and preferring crystalloids over colloids, are depicted in Table 2.

\section{Phase of Fluid Resuscitation and Role of Balanced Solutions}

One third of respondents $(n=17 / 47,36.2 \%)$ chose the fluid depending on the phase of volume resuscitation. Balanced electrolyte solutions were important/very important for the treatment of pediatric shock in two thirds $(n=29 / 47,61.7 \%)$, neither important nor unimportant in one third $(n=13 / 47,27.7 \%)$, and unimportant/ very unimportant in $10.6 \%$ of cases $(n=5 / 47)$.

\section{Blood Products}

Almost all participants ( $n=42 / 43,97.7 \%$, do not sum up to $100 \%$ as multiple answers were possible) administered packed red blood cells $(\mathrm{RBC})$ two-third $(\mathrm{n}=26 / 43,60.5 \%)$ thrombocyte concentrate, 4 in $5(\mathrm{n}=38 / 43,88.4 \%)$ fresh frozen plasma (FFP), and only 1 in $5(n=8 / 43,18.6 \%)$ fibrinogen. Eighteen (41.9\%) of 43 subjects also applied BPs for nonhemorrhagic shock (group A: $n=8 / 16,50.0 \%$; group B: $10 / 27,37.0 \%$ ); septic and hypovolemic shock were the most and second most frequent indication (Tables 3, 4). Thirty-nine percent $(n=17 / 43)$ treated severe head injury (SHI), defined as having glasgow coma scale of less than 9 (group A: $\mathrm{n}=10 / 16,62.5 \%$; group $\mathrm{B}: \mathrm{n}=7 / 27,25.9 \%$ ), and $41.2 \%(\mathrm{n}=7 / 17)$ differentiated in their choice of IV fluid depending on whether or not children with shock did or did not have concomitant SHI (Table 5).

\section{Tranexamic Acid}

One in 3 respondents $(n=14 / 43,32.6 \%)$ administered TXA for hemorrhagic shock (group A: $\mathrm{n}=8 / 16,50.0 \%$; group B: $\mathrm{n}=6$ / $27,22.2 \%$ ) with quantities ranging from 10 to $25 \mathrm{mg} / \mathrm{kg}: 10 \mathrm{mg} / \mathrm{kg}$ $(\mathrm{n}=2 / 14,14.3 \%), 10$ to $15 \mathrm{mg} / \mathrm{kg}(\mathrm{n}=1 / 14,7.1 \%), 15 \mathrm{mg} / \mathrm{kg}(\mathrm{n}=3 /$ $14,21.4 \%), 15$ to $20 \mathrm{mg} / \mathrm{kg}(\mathrm{n}=2 / 14,14.3 \%), 20 \mathrm{mg} / \mathrm{kg}(\mathrm{n}=3 / 14$, $21.4 \%)$, and $25 \mathrm{mg} / \mathrm{kg}(\mathrm{n}=1 / 14,7.1 \%)$.

\section{Massive Transfusions and Protocols}

Half of respondents $(n=22 / 43,51.2 \%)$ performed massive transfusions. However, MTPs only existed in 4 departments,

TABLE 4. Blood Product Used for Nonhemorrhagic Shock

\begin{tabular}{lccc}
\hline Type of Shock & $\begin{array}{c}\text { BP Used for Nonhemorrhagic } \\
\text { Shock }(\mathbf{n}=\mathbf{1 8})\end{array}$ & $\begin{array}{c}\text { Group A, BP Used for } \\
\text { Nonhemorrhagic Shock (n=8) }\end{array}$ & $\begin{array}{c}\text { Group B, BP Used for } \\
\text { Nonhemorrhagic Shock (n= 10) }\end{array}$ \\
\hline Anaphylactic & $1(5.6)$ & $1(12.5)$ & $0(0)$ \\
Septic & $11(61.1)$ & $7(87.5)$ & $4(40.0)$ \\
Cardiogenic & $5(27.8)$ & $4(50.0)$ & $1(10.0)$ \\
Hypovolemic & $9(50.0)$ & $3(37.5)$ & $6(60.0)$ \\
Hypovolemic (within context of DKA) & $1(5.6)$ & $1(12.5)$ & $0(0)$ \\
Neurogenic & $1(5.6)$ & $1(12.5)$ & $1(10.0)$ \\
\hline
\end{tabular}

Values are number (percentage).

Groups do not sum up because multiple answers were possible. 
TABLE 5. Fluids Used in Case of Concomitant SHI and in Case of Absence of Concomitant SHI

\begin{tabular}{lc}
\hline IV fluids used in case of concomitant SHI $(\mathrm{n}=7)$ & $\mathrm{n}=7$ \\
NS & $5(71.4)$ \\
Hypertonic saline & $5(71.4)$ \\
RA & 0 \\
LR & 0 \\
FFP & 0 \\
HA & 0 \\
Gelatine & 0 \\
IV fluids used in case of absence of SHI $(\mathrm{n}=7)$ & \\
NS & $4(57.1)$ \\
Hypertonic saline & 0 \\
RA & $1(14.3)$ \\
LR & $3(42.9)$ \\
FFP & $2(28.6)$ \\
HA & $1(14.3)$ \\
Gelatine & $1(14.3)$ \\
Mannitol & 0 \\
\hline
\end{tabular}

Values are number (percentage).

Groups do not sum up because multiple answers were possible.

primarily in tertiary hospitals (group A: $\mathrm{n}=3 / 16,18.8 \%$; group $\mathrm{B}$ : $\mathrm{n}=1 / 25,4 \%)$.

\section{Differences Between East and West Switzerland}

We observed statistically significant $(P<0.01)$ differences in the choice of IV fluid between the French, including bilingual German-/French-speaking areas (West Switzerland) and German-/ Italian-speaking regions (East Switzerland) (Table 6). West Switzerland exclusively administered NS to prematurely and term newborns and to infants and children in $89.5 \%$ of cases. In contrast, only $60.0 \%(n=15)$ of East Switzerland's respondents exclusively used NS in prematurely and term newborns and $36.4 \%(n=8)$ in infants and children (Table 6).

\section{DISCUSSION}

We observed a distinct clinical practice variation regarding IV fluid administration among Swiss pediatric emergency physicians, pediatric intensivists, and neonatologists. Although pediatric resuscitation guidelines recommend the use of "isotonic crystalloids," 3 there is growing evidence that BES might be preferable to NS for fluid resuscitation except in case of preexisting cerebral edema or chloride deficiency. ${ }^{2,13}$ However, supporting data mainly stem from the adult population ${ }^{9}$ and studies in children are scarce. Our survey reveals this mentioned trend toward the use of BES, however, with a rift between West Switzerland, still preferring NS, and East Switzerland, tending to use BES and to prefer to LR to RA. Although acetate might possess some cardiodepressant and vasodilator effects, it has advantages over lactate, stabilizing $\mathrm{pH}$ more rapidly and being metabolized in various tissues, whereas lactate is processed predominantly in the liver. Bicarbonate, the logical alternative to lactate/acetate, is impracticable because of storage difficulties and its sensitivity to $\mathrm{CO}_{2}{ }^{13} \mathrm{Al}-$ though associated with hyperchloremic metabolic acidosis in adults, ${ }^{2,5,9} \mathrm{NS}$ is the most frequently used IV fluid in all pediatric and neonatal age groups across Switzerland. This may be explained by scarcity of consistent CPGs specifying the choice of IV fluid. Even if only half of respondents differentiated in the choice of IV fluid depending on the presence or absence of concomitant SHI, these $50 \%$ followed the current opinion by using NS/hypertonic saline for patients at risk for brain edema. ${ }^{13}$ The surveyed initial volume administration for shock is in line with current recommendations, ${ }^{3}$ that is, administration of $20 \mathrm{~mL} / \mathrm{kg}$ of isotonic crystalloid boluses, ${ }^{3}$ and smaller volumes $(5-10$ $\mathrm{mL} / \mathrm{kg}$ ) for cardiogenic shock to avoid deterioration of cardiac failure. ${ }^{14}$ Hemorrhagic shock and active bleeding require RBC transfusion not later than after two $20 \mathrm{~mL} / \mathrm{kg}$ of NS boluses. ${ }^{14}$ Subsequent fluid administration should be tailored to the individual with frequent clinical reassessments. ${ }^{3}$ Aggressive fluid resuscitation may be harmful in patients in resource-limited settings, ${ }^{3,16}$ whereas a faster rate of IV rehydration did not significantly influence neurologic outcomes in DKA. ${ }^{15}$ Likewise, the observed colloid application is in accordance with current recommendations, that is, colloid administration for failing preservation of circulation and intravascular volume expansion in septic shock. ${ }^{17}$ Administration of BP for nonhemorrhagic shock has been previously reported $^{11}$ but stands out against recommendations by the British and Italian hematology societies not advising to apply BP as intravascular volume expanders but only for active bleeding and abnormal coagulation tests. ${ }^{18,19}$ Only few studies have examined the ratio of BP administered in children ${ }^{12}$ and only few MTPs exist in Switzerland. However, adherence to MTPs did reduce mortality in adults, but further pediatric studies are needed. ${ }^{20-22} \mathrm{We}$ suggest to implement MTPs in all pediatric institutions to standardize transfusion processes. Our findings reflect a previously observed rare use of TXA in acute pediatric trauma ${ }^{23-25}$ with a wide range of TXA doses. ${ }^{26}$ Additional studies are warranted to define indications for TXA and guidelines for dosage among children, especially in trauma. ${ }^{26}$

\section{Limitations of the Study}

Mixed PED/PICU/NICU and NICUs $(42.5 \%$ of respondents) answered for mixed patient groups, including neonates. We recognize that neonatal patients, however, might require treatment for different pathologies than infants and children. To

TABLE 6. Differences in Choice of IV Fluid Between West (Including Bilingual Regions) and East Switzerland

\begin{tabular}{|c|c|c|c|c|}
\hline & NS & BES & NS or BES & $P$ (Fisher Exact) \\
\hline Prematurely ( $<37$ wk of gestation) and term born infants ( $<28 \mathrm{~d}$ of age) & & & & 0.0033 \\
\hline East $(n=25)$ & $15(60.0)$ & $3(12.0)$ & $7(28.0)$ & \\
\hline West $(\mathrm{n}=19)$ & $19(100)$ & 0 & 0 & \\
\hline Infants ( $>28 \mathrm{~d}$ of age) and children & & & & 0.0014 \\
\hline East $(n=22)$ & $8(36.4)$ & $4(18.2)$ & $10(45.5)$ & \\
\hline West $(\mathrm{n}=19)$ & $17(89.5)$ & 0 & $2(10.5)$ & \\
\hline
\end{tabular}

Values are number (percentage), unless otherwise indicated. 
minimize this bias, we analyzed all not age-specific answers for groups including and excluding neonatal patients separately. Furthermore, we received diverse answers from the same department in 4 cases. This, however, demonstrated inconsistent IV fluid administration practice within 1 department and underlines the need for uniform practice guidelines.

\section{CONCLUSIONS}

Our study reveals distinct clinical practice variations in IV fluid resuscitation in pediatric acute care medicine in Switzerland. Our findings combined with dissention on this topic in the literature and lack of evidence in children warrant future interventional studies determining the benefits and adverse effects of various crystalloids, a better understanding of the pediatric pathophysiology, and new fluid compositions based on future study results. ${ }^{13}$ We suggest the review and implementation of evidence-based CPG to standardize this frequent intervention, minimize complications, and ultimately enhance patient safety.

\section{ACKNOWLEDGMENTS}

The authors thank all respondents for their contribution of answers to this survey.

\section{REFERENCES}

1. Moritz ML, Ayus JC. Intravenous fluid management for the acutely ill child. Curr Opin Pediatr. 2011;23:186-193.

2. Myburgh JA, Mythen MG. Resuscitation fluids. N Engl J Med. 2013;369: 1243-1251.

3. Maconochie IK, de Caen AR, Aickin R, et al. Part 6: pediatric basic life support and pediatric advanced life support: 2015 International Consensus on Cardiopulmonary Resuscitation and Emergency Cardiovascular Care Science with Treatment Recommendations. Resuscitation. 2015;95: e147-e168.

4. Blumberg N, Cholette JM, Pietropaoli AP, et al. $0.9 \% \mathrm{NaCl}$ (normal saline) perhaps not so normal after all? Trans Apher Sci. 2018;57:127-131.

5. Semler MW, Rice TW. Saline is not the first choice for crystalloid resuscitation fluids. Crit Care Med. 2016;44:1541-1544.

6. Allen $\mathrm{CH}$, Goldman $\mathrm{RD}$, Bhatt $\mathrm{S}$, et al. A randomized trial of Plasma-Lyte A and $0.9 \%$ sodium chloride in acute pediatric gastroenteritis. BMC Pediatr. 2016;16:117.

7. Mahajan V, Sajan SS, Sharma A, et al. Ringers lactate vs normal saline for children with acute diarrhea and severe dehydration - a double blind randomized controlled trial. Indian Pediatr. 2012;49:963-968.

8. Jucá CA, Rey LC, Martins CV. Comparison between normal saline and a polyelectrolyte solution for fluid resuscitation in severely dehydrated infants with acute diarrhoea. Ann Trop Paediatr. 2005;25:253-260.
9. Semler MW, Self WH, Wanderer JP, et al. Balanced crystalloids versus saline in critically ill adults. $N$ Engl J Med. 2018;378:829-839.

10. Nevin DG, Brohi K. Permissive hypotension for active haemorrhage in trauma. Anaesthesia. 2017;72:1443-1448.

11. Karam O, Tucci M, Lacroix J, et al. International survey on plasma transfusion practices in critically ill children. Transfusion. 2014;54: 1125-1132.

12. Dehmer JJ, Adamson WT. Massive transfusion and blood product use in the pediatric trauma patient. Semin Pediatr Surg. 2010;19: 286-291.

13. Santi M, Lava SA, Camozzi P, et al. The great fluid debate: saline or so-called "balanced" salt solutions? Ital J Pediatr. 2015;41:47.

14. Löllgen R, Szabo L. Shock in infants and children [in German]. Med Klin Intensivmed Notfmed. 2015;110:338-345.

15. Kuppermann N, Ghetti S, Schunk JE, et al. Clinical trial of fluid infusion rates for pediatric diabetic ketoacidosis. $N$ Engl J Med. 2018;378: 2275-2287.

16. Maitland K, Kiguli S, Opoka RO, et al. Mortality after fluid bolus in African children with severe infection. N Engl J Med. 2011;364: 2483-2495.

17. Rhodes A, Evans LE, Alhazzani W, et al. Surviving Sepsis Campaign: International Guidelines for Management of Sepsis and Septic Shock: 2016. Intensive Care Med. 2017;43:304-377.

18. O'Shaughnessy DF, Atterbury C, Bolton Maggs P, et al. Guidelines for the use of fresh-frozen plasma, cryoprecipitate and cryosupernatant. $\mathrm{Br} J$ Haematol. 2004;126:11-28.

19. Liumbruno G, Bennardello F, Lattanzio A, et al. Recommendations for the transfusion of plasma and platelets. Blood Transfus. 2009;7:132-150.

20. Gilley M, Beno S. Damage control resuscitation in pediatric trauma. Curr Opin Pediatr. 2018;30:338-343.

21. Hwu RS, Spinella PC, Keller MS, et al. The effect of massive transfusion protocol implementation on pediatric trauma care. Transfusion. 2016;56: 2712-2719.

22. Chidester SJ, Williams N, Wang W, et al. A pediatric massive transfusion protocol. J Trauma Acute Care Surg. 2012;73:1273-1277.

23. Faraoni D, Goobie SM. The efficacy of antifibrinolytic drugs in children undergoing noncardiac surgery: a systematic review of the literature. Anesth Analg. 2014;118:628-636.

24. Faraoni D, Willems A, Melot C, et al. Efficacy of tranexamic acid in paediatric cardiac surgery: a systematic review and meta-analysis. Eur $J$ Cardiothorac Surg. 2012;42:781-786.

25. White N, Bayliss S, Moore D. Systematic review of interventions for minimizing perioperative blood transfusion for surgery for craniosynostosis. J Craniofac Surg. 2015;26:26-36.

26. Nishijima DK, Monuteaux MC, Faraoni D, et al. Tranexamic acid use in United States children's hospitals. J Emerg Med. 2016;50:868-874.e861. 